Article

\title{
Multi-Sensor Platform for Indoor Mobile Mapping: System Calibration and Using a Total Station for Indoor Applications
}

\author{
Friedrich Keller * and Harald Sternberg \\ Geomatik, HafenCity Universität, Hebebrandstraße 1, D-22297 Hamburg, Germany \\ E-Mail: harald.sternberg@hcu-hamburg.de \\ * Author to whom correspondence should be addressed; E-Mail: friedrich.keller@hcu-hamburg.de; \\ Tel.: +49-404-2827-5931.
}

Received: 10 September 2013; in revised form: 19 October 2013 / Accepted: 22 October 2013 /

Published: 6 November 2013

\begin{abstract}
This paper addresses the calibration of mobile mapping systems and the feasibility of using a total station as a sensor for indoor mobile mapping systems. For this purpose, the measuring system of HafenCity University in Hamburg is presented and discussed. In the second part of the calibration, the entire system will be described regarding the interaction of laser scanners and other parts of the system. Finally, the preliminary analysis of the use of a total station is presented in conjunction with the measurement system. The difficulty of time synchronization is also discussed. In multiple tests, a comparison was made versus a reference solution based on GNSS. Additionally, the suitability of the total station was also considered for indoor applications.
\end{abstract}

Keywords: calibration; total station; Kalman filter; inertial navigation

\section{Introduction}

In recent years, mobile mapping systems have become a field of research in geodesy. The mobile LiDARsurvey system makes it possible to acquire time-saving and fully-equipped environment data using laser scanners from moving platforms (car, plane, ship). All available mobile mapping systems consist of multiple sensors and can be classified according to two functions: the acquisition of environment data and of trajectories. For the acquisition of the environment, preferably one or more laser scanners are used. These scanners are mounted either perpendicular to the main direction of movement or slightly tilted with two scanners. This latter version is called a butterfly arrangement, because of 
the orientation of the profile planes. For trajectory acquisition, a system can be equipped with three sensors. A GNSSantenna is deployed to provide the absolute position over time. The inertial sensors measure the spatial position and the highly dynamic motion of the system at a very high data rate. The third widely-used sensor is the odometer. An odometer allows precise determination of the traveled distance. Together with the inertial sensor, the odometer is used to compensate for short-term GNSS measurement gaps. Recent studies on MLSsystems and their accuracy can be found in El-Sheimy [1], Kaartinen et al. [2], Brenner [3], Haala et al. [4], Hassan and El-Sheimy [5] or Puente et al. [6] An almost complete overview of the state-of-the-art of mobile mapping systems can be found in Petrie [7] or Graham [8]. Research and development on multisensor mobile mapping systems has been performed since the early 1990s. The development of this system has greatly accelerated in recent years, in part because of technologies, such as Google and its Street View program. One of the latest application trends is to deploy such systems in indoor environments.

If mobile mapping systems are used in buildings, GNSS measurements will no longer be available. As a result, no absolute position can be provided directly by the system itself. This drawback has a serious impact on the inertial measurement unit (IMU), because the pure inertial navigation solutions tend to drift. The magnitude and speed of drift will depend on the quality of the measurement system. This circumstance has led our researchers and developers to consider other aiding sensors and measurement techniques that can substitute the GNSS receivers. At HafenCity University, Hamburg, a modular test platform is being developed that allows for the study of various new aiding sensors.

This platform serves not only as a means to explore new sensors for indoor applications, but also to ensure that a system is suitable for outdoor use, which is the current state of the art. In this paper, the first results using a total station are reported. In addition, the procedure for calibration, which takes place outdoors, will be described.

\section{Measurement System}

To enable the fusion of proven and new sensors, a measurement platform is currently under development that can integrate a variety of sensors. Due to the modular design used, the measuring system can be adapted to face individual challenges. As a research priority, our focus is on the use of a car outdoors and a small trolley indoors. The core sensor in any scenario is always a high-quality IMU sensor (the IMARRQH1003 is used in this research), which enables data recording of various other sensors (odometers and GNSS modules) and the synchronization of all sensors using a clock (pulse-per-second (PPS)). The IMU is topologically the central point of the system, and its body coordinate system can be used to define the platform coordinate system in which the X-axis is in the moving direction of the vehicle, the $\mathrm{Y}$-axis is toward the left and perpendicular to the $\mathrm{X}$-axis, and the $\mathrm{Z}$-axis is perpendicular to both the $\mathrm{X}$ and $\mathrm{Y}$ axes to form a right-handed coordinate frame. The position and the boresight angles of the other sensors can be defined with respect to the IMU body frame. Table 1 shows the deployable hardware modules. The systems may differ according to the working environment or application. Here, the system differs for the car (outdoors) and the trolley (indoors). 
Table 1. Modules of the measurement system.

\begin{tabular}{lcc}
\hline Manufacturer & Name & Accuracy/Resolution \\
\hline \multicolumn{2}{l}{ Inertial Measurement Systems } \\
IMAR & RQH1003 & $0.003 \frac{\mathrm{deg}}{\sqrt{h}}$ \\
\hline GNSS & OEMV-2 & - \\
$\begin{array}{c}\text { Novatel Receiver } \\
\text { Leica }\end{array}$ & AT502 Dual-frequency antenna & - \\
\hline Laserscanner & & \\
Zoller + Fröhlich & 5010 & $0.0004 \mathrm{deg} / 5 \mathrm{~mm}$ \\
$\quad$ Sick & LMC121 & $12 \mathrm{~mm}$ \\
\hline $\begin{array}{l}\text { Odometer } \\
\text { Wachendorff } \\
\text { Peiseler }\end{array}$ & WDG58B & $10,000 \mathrm{I} / \mathrm{U}$ \\
\hline Total station & Kfz-2000 & $2,000 \mathrm{I} / \mathrm{U}$ \\
$\quad$ Leica & TPS1201+ & $0.15 \mathrm{mgon} / 3 \mathrm{~mm}+1.5 \mathrm{ppm}$ \\
\hline Camera & \\
Point Grey & 2x Flea3 stereo & $1.4 \mathrm{MPixel}$ \\
\hline
\end{tabular}

\subsection{Outdoor}

The current configuration for outdoor applications includes an IMU sensor and a GNSS receiver, as is usual for MMS, an approved off-road odometer and a laser scanner. The odometer's position is determined via a calibration process using a total station. The data recording and synchronization of the individual sensor modules are automatically controlled by the IMU. The GNSS module is linked to the IMU through the lever arm of the antennas, which can be determined with respect to the IMU center using a total station. The GNSS data are stored by the IMU. In addition, the PPS from the GNSS is used to stabilize the timing system. The PPS can be passed on to the other sensors by the IMU. The commonly used laser scanners from Zoller + Fröhlich allow for two different types of temporal synchronization. Therefore, it is important to know that the scanner saves its data line by line. One rotation of the scanner's head is a line, and each measured point corresponds to a pixel in this line. In a variant of the synchronization, the PPS is passed to the scanner. The scanner then marks the corresponding point in the relevant line by a flag at the time the PPS arrives. This procedure is relatively complicated, because each line must be searched for appropriate flags.

The second option is much easier. The scanner itself generates a pulse for each complete rotation of the scanner's head. This pulse can be transferred to the IMU and is chronologically referenced and stored. Thus, each line can be assigned a start time tag and an end time tag. The position and orientation of a laser scanner in the vehicle coordinate system is discussed in Section 3. This calibration is always performed with the outdoor configuration, because it allows for long distance measurements.

The plan also includes a camera module for various applications, such as the detection of lane markers.

\subsection{Indoors}

The indoor configuration, Figure 1b, contains the main IMU module, a Wachendorff odometer and a laser scanner. Because the GNSS module cannot be used indoors, several other modules can be integrated 
to combat the drift of the IMU. The integration of the additional aiding sensors is currently being planned or has already been implemented and evaluated for suitability. One choice is to use the stereo camera as an aiding sensor. A horizontal measuring laser scanner by SICKusing the simultaneous localization and mapping (SLAM) method can also be used.

Figure 1. The mobile mapping system; the left shows the system mounted on a van outdoors; the right shows the indoor set-up, where the GNSS-antenna can be replaced with a prism. (a) Outdoor configuration; (b) indoor configuration.

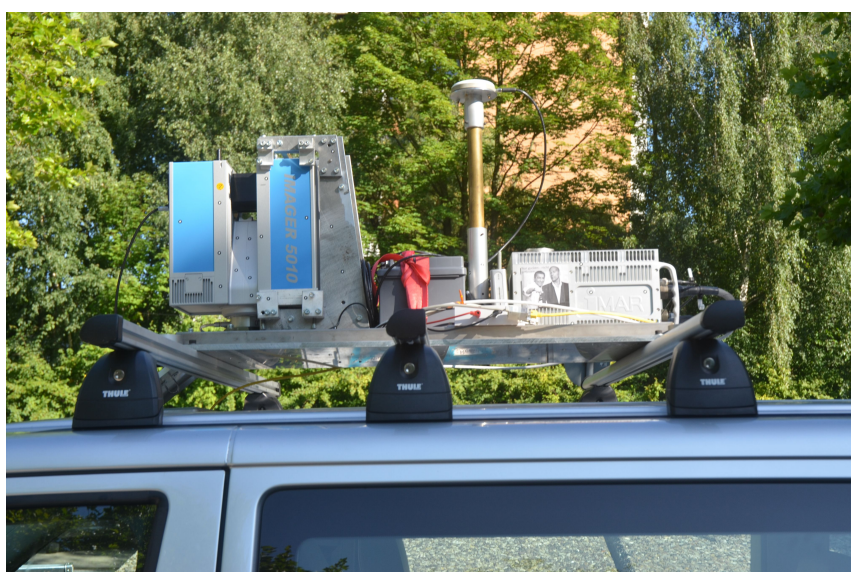

(a)

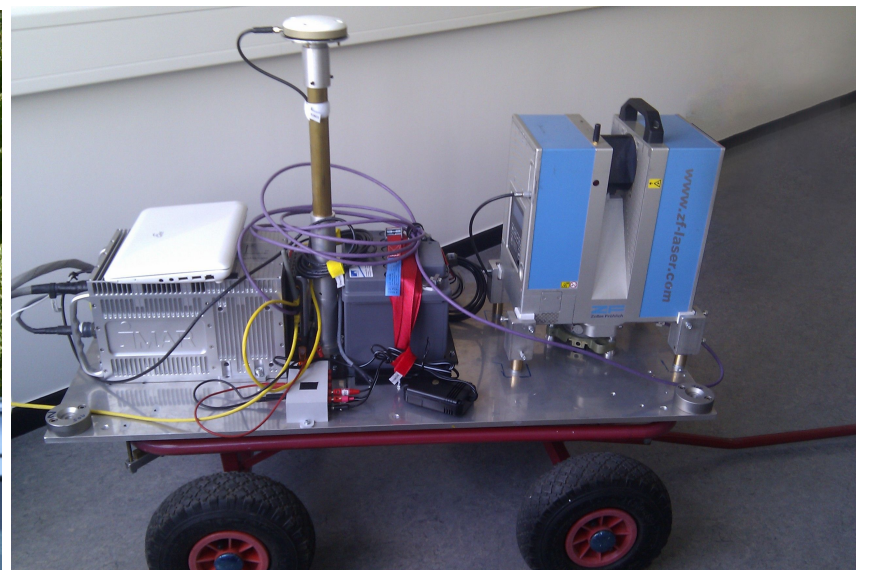

(b)

Because only one high-end laser scanner is available, it is mounted perpendicular to the direction of travel. The possibilities of the system should be explored and demonstrated in this project. For very economical use, two scanners in butterfly mode should be used.

The use of SLAM appears promising, which is not only due to the many developments in the field of robotics, but also to the combination of two laser scanners, one low-cost and one high-end. These sensors are used to determine the position and to capture the environment. This configuration option is still at the conceptual stage.

The use of a total station is currently being investigated as another alternative. In this system, a $360^{\circ}$ degree prism is mounted to the platform with its position predetermined in the platform coordinate system. The prism is followed by a total station in the tracking mode during the movement of the platform. A total station, as one of the most precise geodetic instruments, can be set up at various known positions along with the known orientations, so that it always has an optimal view of the platform. The total station can function in the same way as a GNSS receiver. The first results of the investigation are shown in Sections 4 and 6.

\section{Calibration}

This section discusses the calibration of the whole system and the interaction of environment and trajectory acquisition. Because the platform coordinate system is identical to the IMU body coordinate system, the positions of most modules can be determined in this coordinate system using their lever-arms to the IMU. However, three boresight angles are needed for a laser scanner as a 3D object with respect to the IMU body. The calibration determines the position and attitude of the four-dimensional coordinate 
system of the laser scanner (x, y, z, t) (shown in blue in Figure 2) with respect to the four-dimensional IMU platform system (shown in red in the Figure 2). The position of the anchor point of the blue system to the reference system of the IMU can be described by a lever arm with $\Delta x, \Delta y$ and $\Delta z$. The orientation, i.e., the rotation of the axes, is given by the angles, $\phi, \theta$ and $\psi$. Thus, $\phi$ is the rotation around the $\mathrm{X}$-axis of the IMU, $\theta$ around the Y-axis and $\psi$ around the $\mathrm{Z}$-axis. The fourth dimension is time. For this purpose, the time systems of the laser scanner and the IMU must be synchronized.

Figure 2. Schematic diagram of the measuring system with the two main components of the laser scanner and the inertial measurement unit (IMU) sensor. The coordinate system of the IMU is drawn in red and defines the body coordinate system of the platform. The location of the blue laser scanner coordinate system must be known in the red system.

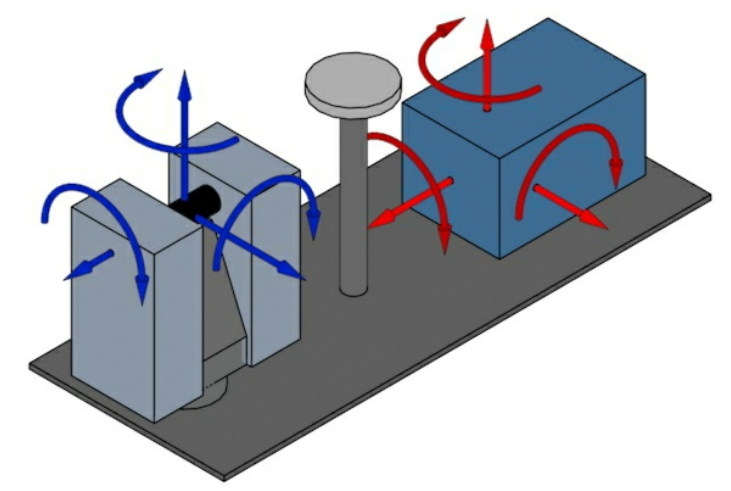

\subsection{Estimation of the Accuracy}

To decide with what accuracy a calibration should be performed, certain considerations are necessary. If it is assumed that the trajectory would be error-free, then only the accuracy of the laser scanner and its lever arm and boresight angles are decisive. The laser scanner has an angular accuracy of a few ten-thousandths of a degree and a distance measurement accuracy of a few millimeters (Table 1). These are the criteria for the required accuracy of a calibration. Of course, the trajectory is not error-free. Because the accuracy of the trajectory is approximately two to three centimeters [9], the calibration accuracy needs to be far less than the trajectory accuracy to avoid significantly influencing the final result. Theoretically, it should be below the accuracy of the scanner, but this is considered uneconomical, due to the time required and the unknown stability of the calibration. The geometrical considerations or variance propagation demonstrate that a determination of the translation $\Delta x, \Delta y$ and $\Delta z$ is enough for one to two millimeters. The angles, $\phi, \theta$ and $\psi$, should be determined to approximately $0.005^{\circ}$ degrees, which corresponds to an accuracy of $4 \mathrm{~mm}$ for a typical measurement distance of $50 \mathrm{~m}$ meters in an urban area. For the temporal reference, an accuracy of $0.1 \mathrm{~ms}$ is desired, which results in a $2 \mathrm{~mm}$ deviation at a speed of $72 \mathrm{~km} / \mathrm{h} \mathrm{[10].} \mathrm{Various} \mathrm{methods} \mathrm{can} \mathrm{be} \mathrm{used} \mathrm{to} \mathrm{satisfy} \mathrm{the} \mathrm{accuracy} \mathrm{requirement.} \mathrm{These} \mathrm{methods}$ are presented in the following section.

\subsection{Internal Position and Orientation Determination}

For internal position and orientation determination, the scanner itself can be used as a measuring system. A sufficient number of targets must be attached to the platform and spatially distributed 
to maximize the system calibration. Their positions are determined using a total station or the photogrammetric method in the platform coordinate system. The scanner can detect the targets by a simple 360-degree scan. The conformal transformation process between two different coordinates of each target will deliver the parameters, i.e., the lever arm and boresight angles of the laser scanner. The targets can be spherical or black and white targets. The scanner has to be rigidly connected to the platform to avoid suffering any possible rotation.

\subsection{External Position and Orientation Determination}

The external position and orientation determination includes all of the procedures determined by the lever arm, an additional measuring system and the position angle. Various instruments can be used for this purpose, such as total stations, photogrammetry, fringe projection systems or laser trackers. For the platform at the HCU, a fringe projection system is used, because its size is only $1 \mathrm{~m}$ by $0.4 \mathrm{~m}$. Whether these instruments are suitable for the position and orientation determination in terms of accuracy will be discussed through two examples. In the first example, it is assumed that the lever arm of the laser scanner $\Delta x, \Delta y$ or $\Delta z$ was determined at an accuracy of $1 \mathrm{~mm}$ and that the vehicle moves on a road that has a slope of $4^{\circ}$ degrees in the traveling direction. The Figure 3 shows that the correct lever arm, $T L S 0$, leads to a fault point, $T L S 0^{\prime}$, due to the error, $H$. With a slope of $\alpha$, the points shift toward $T L S$ and $T L S^{\prime}$.

$$
\begin{aligned}
& S=H \cdot \sin (\alpha) \\
& C=H \cdot \cos (\alpha)
\end{aligned}
$$

Figure 3. Impact of a specifically biased lever arm; when the lever arm is incorrectly determined by the amount of $H$ and the system is rotated by the angle, $\alpha$, the wrong lever arm can result in an error of $C$ and $S$, causing the actual measured point, $P$, to be at the wrong point, $P^{\prime}$.

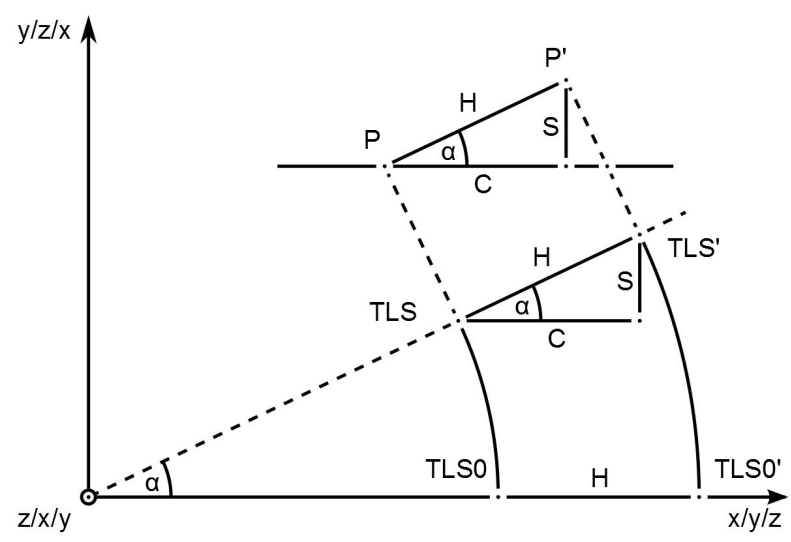

This relation can also be described by Equations (1) and (2). This results in an error for Sand C, respectively, which can be ignored. The second example illustrates the error effect of the boresight angles. It is assumed that the scanner has a measurement deviation of $4 \mathrm{~mm}$ at a distance of $50 \mathrm{~m}$. This results in an angular error of $0.005^{\circ}$. As clarified in Section 3.1, this is the expected accuracy for the 
calibration. To determine the installation angles on the platform, only the body of the scanner can be used. The outside dimensions of the Zoller+ Fröhlich laser scanner are approximately $286 \mathrm{~mm}$. An angle change of $0.005^{\circ}$ on a short basis can cause a deviation of $24 \mu \mathrm{m}$. This deviation is difficult to determine, even using a laser tracker. Furthermore, it should be noted that only the position of the laser scanner's housing on the platform can be determined, not the required orientation of the mirror in the laser scanner body. The external determination for the lever arm, therefore, should be considered. Determination of the angles, however, can be difficult using this method.

\subsection{Field Procedure}

The field procedure is similar to the procedure with the calibration method for multibeam sonar systems [11,12]. With the multi-sensor system, static objects (buildings) are scanned. The faulty installation angles produce typical errors in the point cloud. In the following, possible errors on the roll, pitch and yaw angles will be discussed. A false roll angle generates a point cloud of a tilting facade, which was supposed to be vertical. If the measuring system passes twice from the opposite direction to the facade, the first facade tilts toward the vehicle, and the second facade tilts away from the vehicle. The angle between the two planes corresponds to the double roll angle error and can be determined. For an error-free point cloud, the measurement results have to be averaged (Figure 4a). The situation is similar with an error on the pitch angle, which causes an incorrect installation angle of the tilting facade edges. The facade edge tilts in the traveling direction or in the opposite direction. To determine this error, a straight line is placed in the edge each time. The angle between the two straight lines corresponds to the double pitch angle error (Figure 4b).

Figure 4. Configuration for the calibration of pitch and roll angles; for both angles, when the vehicle passes along an object, the difference of the two passes can be used to estimate the roll and pitch angles. (a) Roll; (b) pitch.

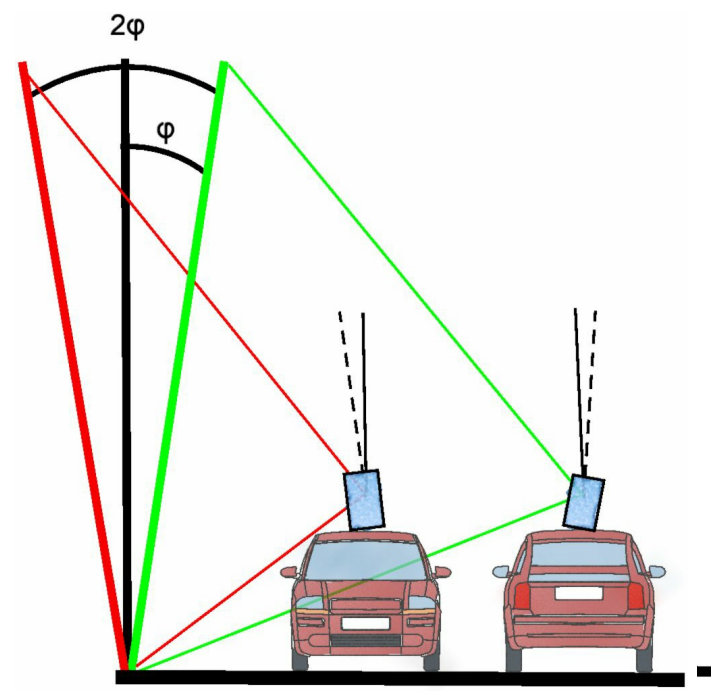

(a)

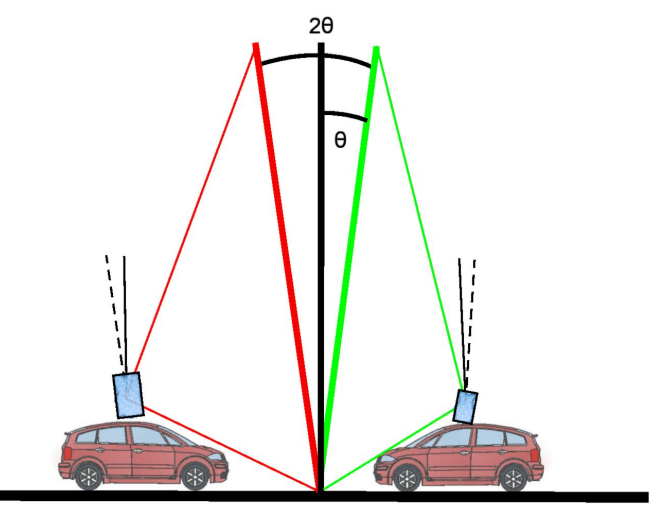

(b)

The residual error on the azimuth can be determined by passing by and surveying a circular object from two sides. As a result, an offset to the actual position of the object is determined. From the distance 
between the two trajectories and the distance between the two circular objects, the angle correction is given (Equation (3)) (Figure 5b).

$$
\delta \alpha=\arctan \left(\frac{\Delta x}{\Delta L}\right)
$$

To determine the residual time error with the synchronization of the laser scanner with respect to the system, the same trajectory is traversed twice at different, but constant, speeds. This eliminates the error effect, due to the installation angles (Equation (4)), because they affect the traveled distance in the same way (Figure 5a).

$$
\delta t=\frac{\Delta x}{v_{2}-v_{1}}
$$

Figure 5. Configuration for the calibration of time offset and yaw angle offset. The vehicle is driven by an object at two different speeds to observe the timing error. The timing error is then derived from the geometric errors. To determine the azimuth error, a round object should be passed by from two sides. The offset between the two objects is then used to estimate the azimuth error. (a) Time; (b) yaw.

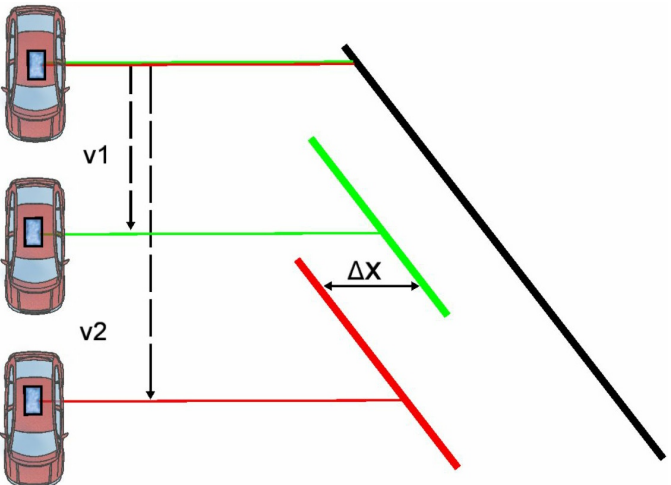

(a)

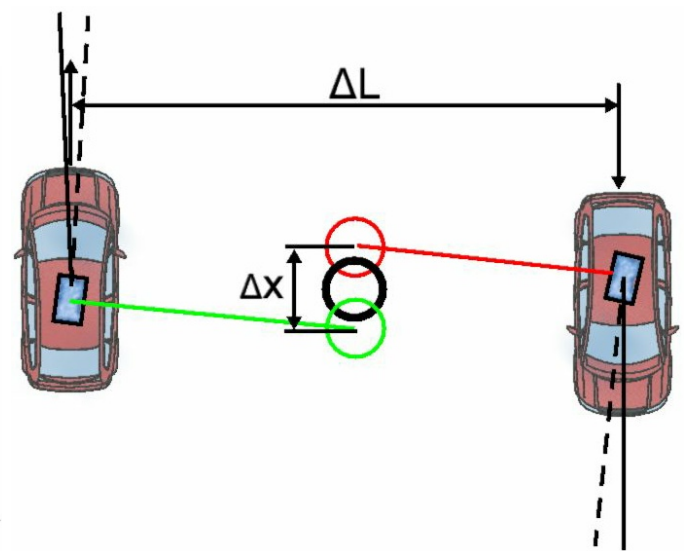

(b)

In summary, a combination of the external determination of the position and the orientation through the field procedure constitutes the most useful method. The calibration provides the lever arm, while the field procedure provides the angles and the time. A disadvantage of the field procedure is that it is not independent on the determination of the trajectory. In addition, significant latency can be determined through the field procedure. Another method to observe the latency between the laser scanner and the IMU was described in [13]. For this purpose, the measurement system has to be rotated around its axis uniformly and in both directions. This results in a shift for a distant sphere from both the right and left sides of the sphere. The latency can be calculated from the difference of the two sphere centers. Whether this type of latency calibration comes into question is still under investigation.

\section{Total Station}

To process the data with the outdoor configuration, a commercial software product called the Inertial Explorer, by Novatel, is used. However, this software is unsuitable for determining the position and 
orientation of the platform indoors. Furthermore, there is no software utility available that can combine all already planned and implemented sensors. Accordingly, an in-house software utility was developed with a Kalman filter as its core. Its main features will be discussed in Section 5. One of the first modules in this software was for the total station. The total station system used was the Leica TPS1201+ with a $360^{\circ}$ degree prism as a target and an adapter to mount the prism to the platform. To make the use of the Kalman filter with kinematic measurement applications convenient, a uniform time base is given. Normally, this time system is defined by the GNSS PPS signal. Because the total station cannot be connected to the IMU and has no trigger capability, an alternate solution must be found for the indoor system. Obviously, the IMU time system allows all connected systems to be referenced in a timely manner. The IMU time system can be synchronized with the GPS time outdoors. If this synchronization cannot be realized, the system time will drift slowly. This error does not have further effects, because all sensors are synchronized via the IMU time. Furthermore, the total station has a time system that differs from the system platform. The time system for the total station was considered in [14]. To synchronize the sensors with little effort using an additional laptop or special measurement configuration, the following linear relation is proposed.

$$
k_{T P S}=m \cdot k_{I M U}+b
$$

where $k_{I M U}$ and $k_{T P S}$ are the same points in time, $m$ is a scale that is generated by the different time drifts of the system and $b$ is the offset between the two time systems. First, it is assumed that both systems have only one offset and a stable scale $m=1$. To determine the offset, $b$, two velocity profiles are needed. The first can be the velocity profile of the odometer. The second can be calculated from the coordinates based on the measurements by the total station. The time offset of the two time systems can be determined using the cross-correlation. However, residual errors will always remain. At the same time, it is not known which time stamp used is associated with the measurements taken by using the total station. In practice, the angle reading takes place immediately, while the distance measurement requires a few milliseconds. This circumstance leads to an incorrect position in kinematic application in terms of time using the total station. Distance and angle measurements cannot be tagged using the same instant of time. The manufacturers have overcome this difficulty. Leica calls this method "Syncrotrack". However, it remains undetermined in this research which time stamp is ultimately stored for the total station measurements.

\section{Sensor Fusion}

To integrate all the measurement data for an optimal trajectory, the extended Kalman filter (EKF) ([15]) was chosen. The Kalman filter algorithm consists of a set of equations realized in two steps: prediction (time update) and correction (measurement update) [16].

\subsection{Prediction}

The predicted state vector is given as follows:

$$
x_{k+1, k}=f\left(x_{k, k}, w_{k}\right)
$$


in which the state vector consists of the following values:

$$
\hat{x}_{k, k}=[\phi, \omega, p, v, a]^{T}
$$

where $\phi$ is the attitude angle vector, $\omega$ is the $3 \mathrm{D}$ angular velocity vector, $p$ is the $3 \mathrm{D}$ position vector, $v$ is the $3 \mathrm{D}$ velocity vector and $a$ is the $3 \mathrm{D}$ acceleration vector. The matrix, $Q$, in Equation (6) models the process noise. $f\left(x_{k, k}\right)$ is a nonlinear function that models the motion. After the linearization by Taylor expansion, Equation (7) becomes:

$$
\hat{x}_{k+1, k}=A_{k, k} \cdot \hat{x}_{k, k}+W w_{k}
$$

in which the term, $A$, is the system transition matrix, $W$ is the Jacobian matrix of partial derivatives of $f$ with respect to $\mathrm{w}$ and $w$-ist, the process noise. To compute the position rotation matrices $\left(C_{x}, C_{y}, C_{z}\right)$ with time-dependent angles, the measured velocities and accelerations in the platform coordinate system are transformed into a superordinate reference system. The rotation matrices are as follows:

$$
\begin{aligned}
C_{x} & =\left(\begin{array}{ccc}
1 & 0 & 0 \\
0 & \cos (\phi) & -\sin (\phi) \\
0 & \sin (\phi) & \cos (\phi)
\end{array}\right) \\
C_{y} & =\left(\begin{array}{ccc}
\cos (\theta) & 0 & \sin (\theta) \\
0 & 1 & 0 \\
-\sin (\theta) & 0 & \cos (\theta)
\end{array}\right) \\
C_{z} & =\left(\begin{array}{ccc}
\cos (\psi) & -\sin (\psi) & 0 \\
\sin (\psi) & \cos (\psi) & 0 \\
0 & 0 & 1
\end{array}\right)
\end{aligned}
$$

where the indices, $x, y$ and $z$, represent which axis each rotation is centered on and $\phi, \theta$ and $\psi$ are the rotation angles. In compliance with the rotation sequence, the total rotation matrix is given by:

$$
C=C_{z} \cdot C_{y} \cdot C_{x}
$$

The transition matrix for the Kalman filter can be described by:

$$
A=\left(\begin{array}{ccccc}
1 & 1 \cdot d t & 0 & 0 & 0 \\
0 & 1 & 0 & 0 & 0 \\
0 & 0 & 1 & C \cdot d t & \frac{1}{2} C \cdot d t^{2} \\
0 & 0 & 0 & 1 & 1 \cdot d t \\
0 & 0 & 0 & 0 & 1
\end{array}\right)
$$

\subsection{Correction}

The measurement equation can generally be described as follows:

$$
z_{k, k}=h\left(x_{k, k}, v_{k, k}\right)
$$

The equation for the linearization of $h\left(x_{k, k}, v_{k, k}\right)$ to $H$ is given as: 


$$
\tilde{z}_{k, k}=H \hat{x}_{k, k}+V v_{k}
$$

in which the term, $H$, is the Jacobian matrix of partial derivatives of $h$ with respect to $x, V$ is the Jacobian matrix of partial derivatives of $h$ with respect to $v, v$ is the measurement noise and $z$ is the measurement. For the available measurement data from the IMU (angular velocities and accelerations) and velocities from the odometer, the corresponding design matrix, $H$, is as follows:

$$
H_{I M U}=\left(\begin{array}{lllllll}
0 & 1 & 0 & 0 & & 0 \\
0 & 0 & 0 & {\left[\begin{array}{lll}
1 & 0 & 0
\end{array}\right]} & 0 \\
0 & 0 & 0 & & 0 & & 1
\end{array}\right)
$$

with three angular velocities, one velocity in the direction of travel and three accelerations; so $H_{I M U}$ is a matrix with $7 \times 15$. Because the measurements from the IMU and the total station are available at different times, a second matrix, $H_{T P S}(3 \times 15)$, for the coordinates determined by the total station is given as:

$$
H_{T P S}=\left(\begin{array}{lllll}
0 & 0 & 1 & 0 & 0
\end{array}\right)
$$

The final solution from the Kalman filter is:

$$
\hat{x}_{k, k}=\hat{x}_{k, k}+K_{k, k}\left(\tilde{z}_{k, k}-H \hat{x}_{k, k}\right)
$$

where $K_{k, k}$ is the Kalman-Gain-Matrix. For a more detailed description, refer to [16].

\subsection{Smoothing}

To improve the filtering results, a Rauch- Tung-Striebel smoother ([17]) is applied. This fixed-interval two-pass implementation is used to provide the fastest fixed-interval smoother. The first (forward) pass uses a Kalman filter, but saves the intermediate results $x_{k, k}, i x_{k+1, k}, P_{k, k}$ and $P_{k+1, k}$ at each measurement time, $k$. The second pass runs backward in time in a sequence from the time of the last measurement, computing the smoothed state estimate from the intermediate results stored on the forward pass. The new smoothed stat vector is obtained recursively [18]:

$$
x_{k, n}=\hat{x}_{k, k}+D_{k}\left(\hat{x}_{k+1, n}-\hat{x}_{k+1, k}\right)
$$

with:

$$
D_{k}=P_{k, k}+F_{k}^{T} P_{k+1, k}^{-1}
$$

\section{Results and Discussion}

The first results of the use of a total station as a sensor module are presented here. Two scenarios were tested, one indoors and one outdoors. Multiple experiments were performed indoors under real 
application conditions. To obtain an independent review, a second scenario was investigated outdoors with the outdoor configuration.

\subsection{Outdoor Test}

The independent review of the total station solution requires measurements under excellent GNSS conditions. The position of the total station was pre-determined using known points in a WGS84/UTM-system using a free station approach, which is necessary to link the existing coordinates of the trajectory to this system and also shows the advantage of using the total station module.

The commercial software product, Novatel Waypoint Inertial Explorer, was used to integrate the GNSS data with the IMU and the odometer data to deliver the best estimate of the trajectory. Figure 6a shows the top view of the trajectory. During the test drive, the platform was tracked by the total station. The (actual trajectory) was created according to the Kalman filter (Section 5) with the aid of the coordinates derived from the total station measurements with the same IMU and odometer data. The timing offset between the total station and the GNSS system was determined using a correlation of the velocity profiler (as in Section 4). Figure $6 \mathrm{~b}$ shows the correlation and the velocity profiles of both the odometer (blue) and the total station (red) after the correction of the time offset. The displacement was $57.188 \mathrm{~s}$ at a correlation of $98 \%$. Figure 7 shows a comparison of the position coordinates, the difference between the nominal (GNSS) and actual (total station) position. Clearly, a large variation existed at the beginning, during the stationary period, which must have resulted from the GNSS measurement.

Figure 6. (a). The traveled trajectory with the positions of the receiver and the total station. Due to the spatial conditions, the trajectory is slightly elongated. (b). The corresponding velocity profiles mapped by the odometer and the total station are shown in the right bottom figure of (b). The cross-correlation of the two velocities is shown in the top-right figure of (b).

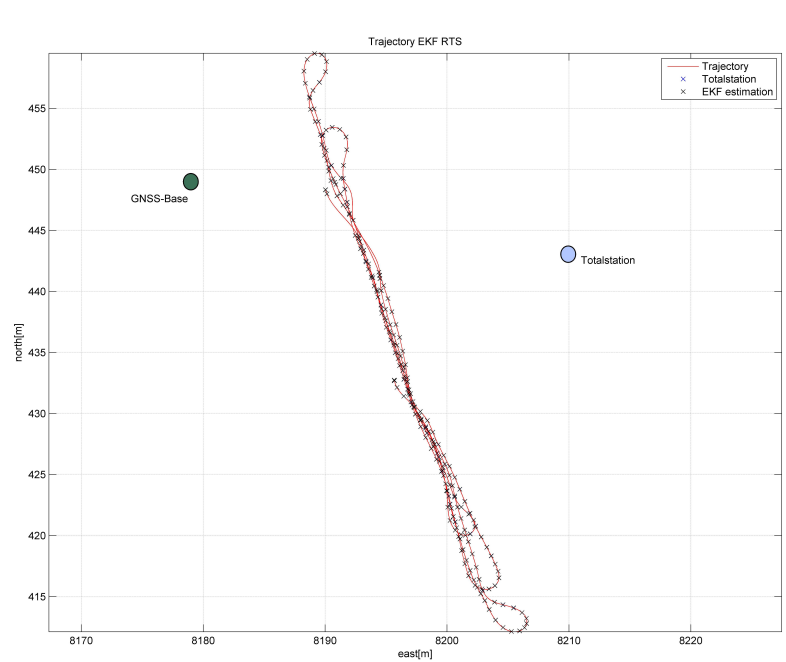

(a)Trajectory
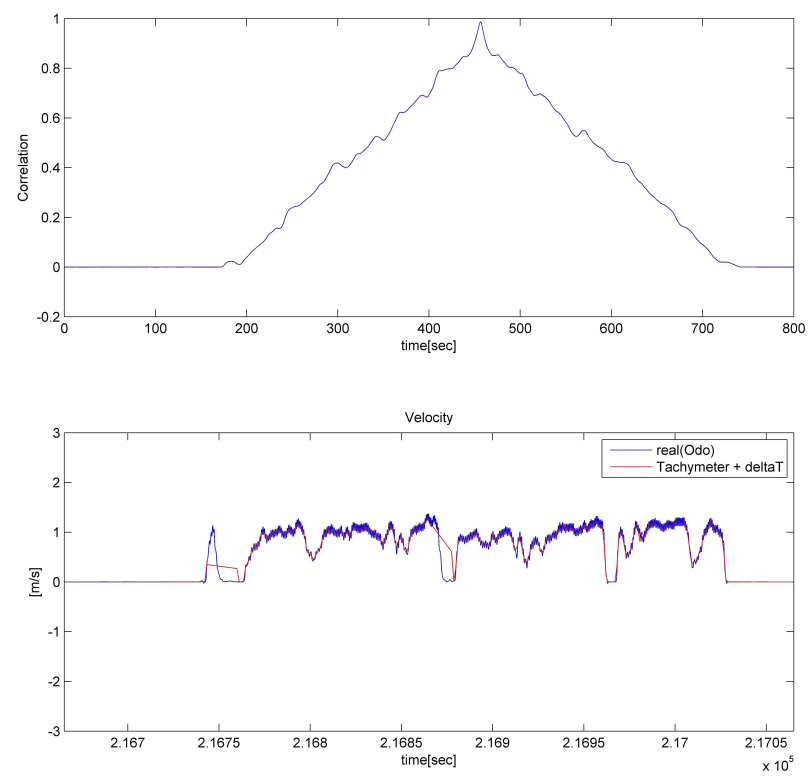

(b)Velocityprofiles and Correlation 
As a result, the review by GNSS must be assessed carefully. If the variation during standstill is taken as the measurement accuracy $(2 \mathrm{~cm}(95 \%))$, only a few significant deviations can be detected. Conversely, this means that the total station can achieve the same accuracy. Moreover, the total station improves the kinematic measurements with IMU. However, the review of the results is difficult, as the GNSS type used was insufficient. Other possibilities, such as the use of a laser tracker, need to be analyzed. The authors of [19] demonstrated a trajectory verification using a Leica AT901 laser tracker and described how the non-existent 360 degree reflector can be replaced by observation from above. The recent developments of the AT901as a long-range system ( $80 \mathrm{~m}$ radius) would make it possible to use a much more accurate measurement system as a reference.

Figure 7. Differences between the Kalman filter solution with the IMU and total station and the solution with GPS and IMU. The variations in the east and north are presented in red and blue, respectively. The temporal resolution is one second. The cyan area represents the GPS variance $(95 \%)$ calculated by the Novatel Inertial Explorer.

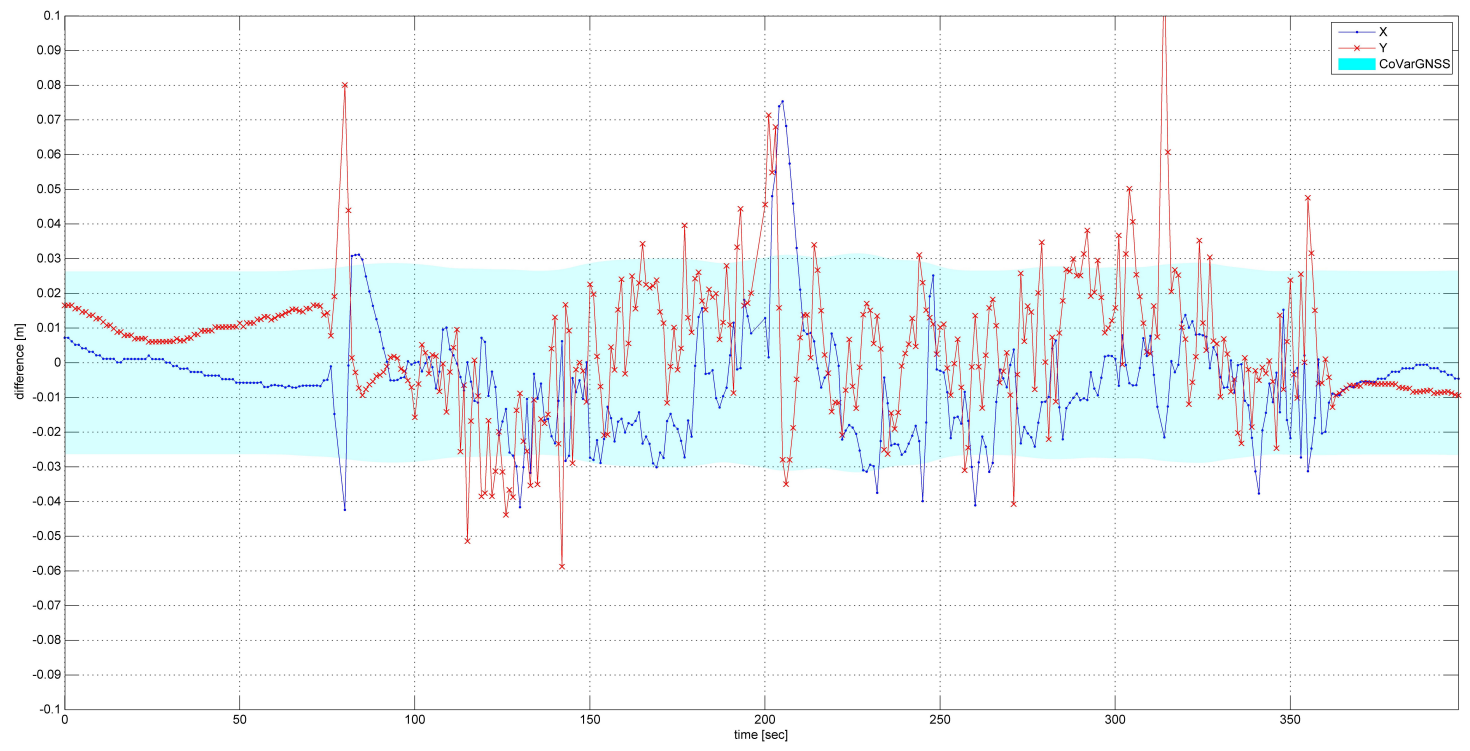

It must also be examined whether the use of GeoCom-commands via a serial interface can enhance time allocation. The detailed analysis of the smoothed trajectory suggests that the estimated offset is not quite constant. Again, the activation of the GeoCom-interface might be helpful.

\subsection{Indoor Test}

To investigate the usefulness of the measurement system indoors, several tests were performed within an HCU building. The indoor area was different in some aspects from the outdoor area. The space was limited, so the total station was operated at close range. Furthermore, there was not always a clear view of the target because of objects, such as railings and plants. One advantage was the level floor, resulting in less vibrations than the ground outdoors. Figure 8 presents two different trajectories. In Figure 8a, the hallway was surrounded. The observation of the total station was therefore interrupted by a large obstruction. Figure $8 \mathrm{~b}$ shows the second obstruction, in which the measurement platform had been moved. In this case, an attempt was made to interrupt the observation as little as possible. 
Figure 8. The two measured indoor trajectories. On the left, a round-trip through the HCUbuilding, with the total station's line of sight interrupted by a visual obstruction. The right image shows a trip without any interruption of the total station observation. (a) Trajectory 1; (b) trajectory 2.

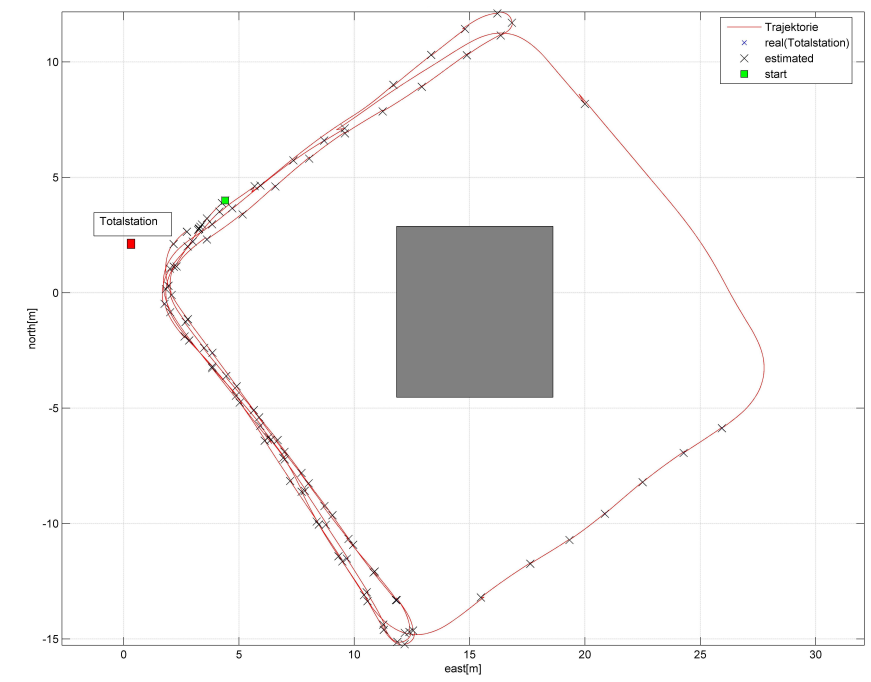

(a)

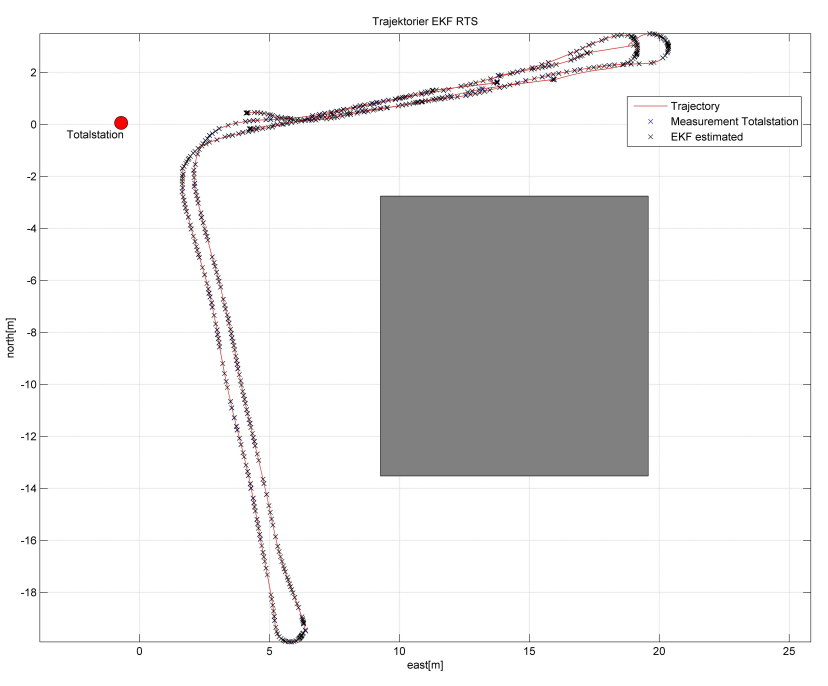

(b)

First, trajectory 1 in Figure 8 a should be examined more closely. The storage times of the total station were used as time stamps, with an interval of one second. After the completion of the measurements, the time offset of the system was determined by cross-correlation as $7.645 \mathrm{~s}$ at a correlation of $96 \%$. These values are variable, as they are dependent on the activation time of the systems. Therefore, the time offset for each measurement has to be redefined. Because GNSS is not an option as an absolute time system in an indoor environment, it is referenced only to the clock of the IMU. After the time systems were aligned to the same time base, only every second point of the total station was used as a control point. These control points are not taken into consideration in the Kalman filter. Thus, the estimated positions from the filter were compared with the control points to perform the analysis of their achievable accuracy, which is summarized in Table 2. Figure 9 shows the coordinate differences in the $\mathrm{X}$ and $\mathrm{Y}$ directions with all subsidies in Case 3. The control points are correlated with the measurement system over time. It can be concluded that an incorrect time alignment is reflected in the positional errors. Various supporting data were tested for the filter. In addition to the IMU, the odometer and the total station were added sequentially, but all other parameters remained unchanged, including the initialization of the filter, which was stationary because of the total station.

Table 2. Error analysis of control points with quadratic deviation; Case 1: IMU; Case 2: IMU and odometry; Case 3: IMU, odometry and total station.

\begin{tabular}{ccccc}
\hline & IMU only $(\mathbf{m})$ & with Odometry $(\mathbf{m})$ & with Total Station $(\mathbf{m})$ & vs. GPS \\
\hline Maximum & 1.8049 & 1.0395 & 0.1105 & 0.1274 \\
Median & 0.5820 & 0.3783 & 0.0101 & 0.0164 \\
Mean & 0.7218 & 0.4140 & 0.0159 & 0.0202 \\
Std. & 0.4872 & 0.3268 & 0.0197 & 0.0149 \\
\hline
\end{tabular}


Figure 9. Differences between the control points of the total station without the GEOCOMconnection and the solution from the Kalman filter with trajectory Case 3.

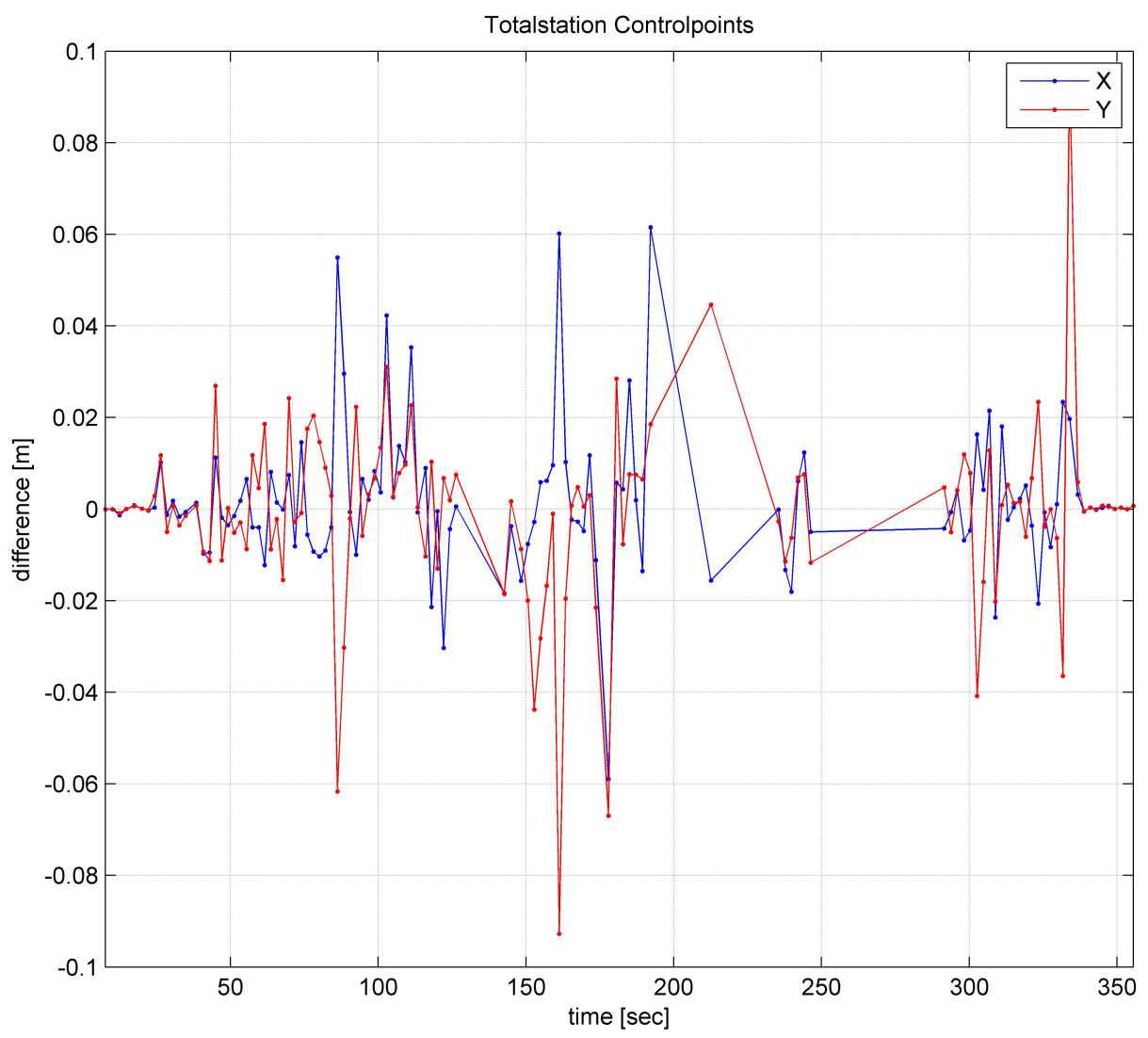

The deviations shown in Table 2 have to be discussed for Cases 2 and 3. It is clear that the mean value from Case 2 is higher than the standard deviation. This result indicates a systematic error in the measurements, which can be explained by the drift of the system. The evenly driven trajectory became larger during the course of the measurements.

The mean value of Case 3 is smaller than the standard deviation. It seems that the systematic error components decreased through the addition of the total station. The residual errors were characterized as random errors, and this point remains the subject of further investigation. To avoid being dependent on an uncertain time stamp for the data storage, a small Java application was created to send measurement commands via the GeoCom interface to the total station. The command:

\section{$\backslash \frac{0}{R} 1 Q, 2167$}

provides a time tagged measurement, which is used to reference the exact time of the measurements. Control via Geo-Comhas the advantage that each measurement can be provided with a code that allows conclusions to be drawn about the measurement's quality. Figure 10 shows trajectory 2 once more. All measurements were color-coded. Code zero indicates that the measurement was "OK". Code 1284 indicates that the total station could not satisfy the usual accuracy [20]. This seems to be a problem, especially at close range. A possible explanation could be that the prism fills the field of view of the sensor chip for positioning, which would complicate the median point determination. A further problem seems to be visual interruptions. Even after a short time, the accuracy could not be ensured after recovery. 
Figure 10. Trajectory 2: all measured points are instantly highlighted by their indexes returned from the total station $(0=\mathrm{OK} ; 1,284=$ accuracy cannot be guaranteed $)$. As expected, code 1284 shows a close range and signal breaks.

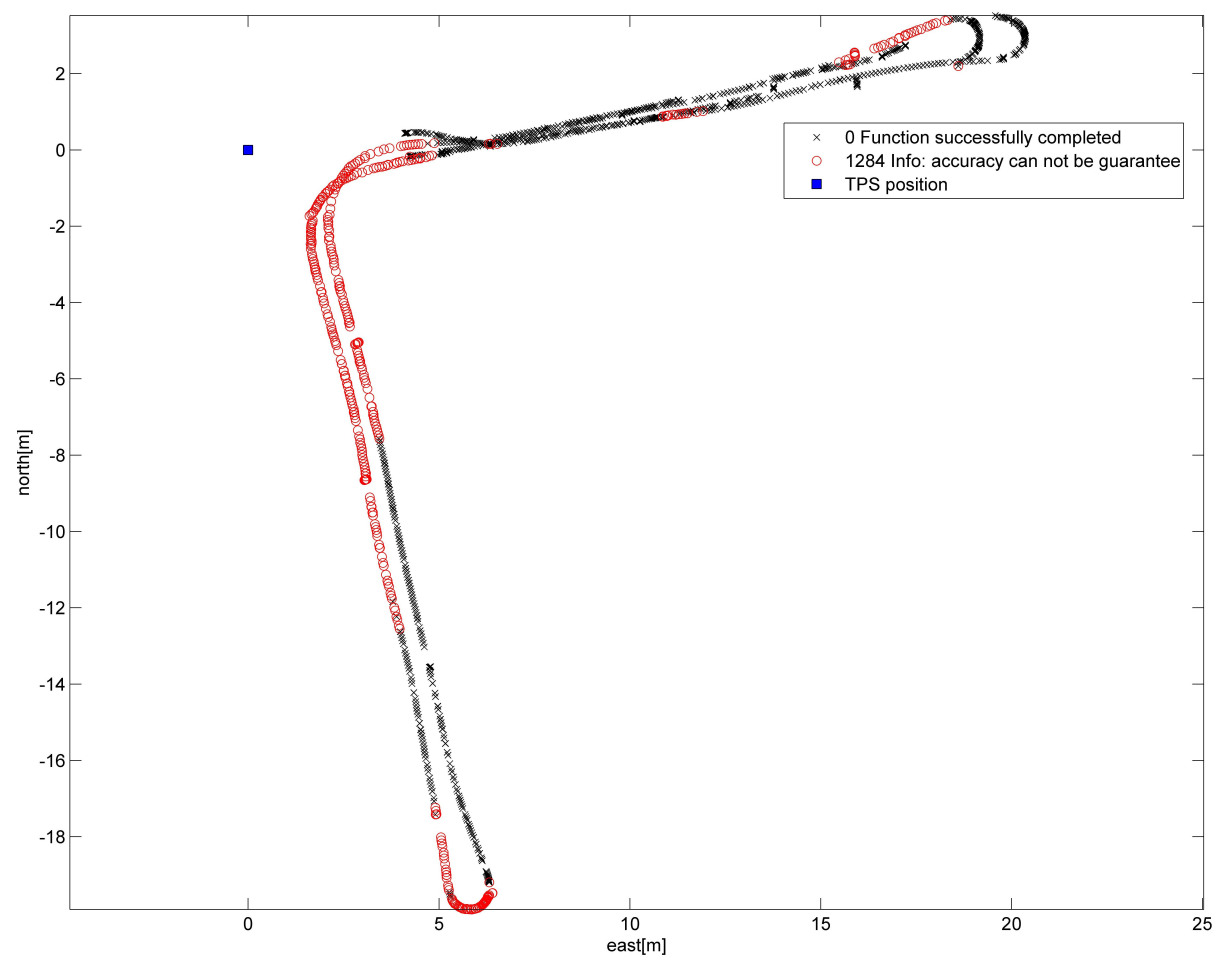

Figure 11. (Top) The correlation with outliers in blue and without outliers in red; (Bottom) The velocity profiles of trajectory 2 ; the odometer in blue and the total station in green.
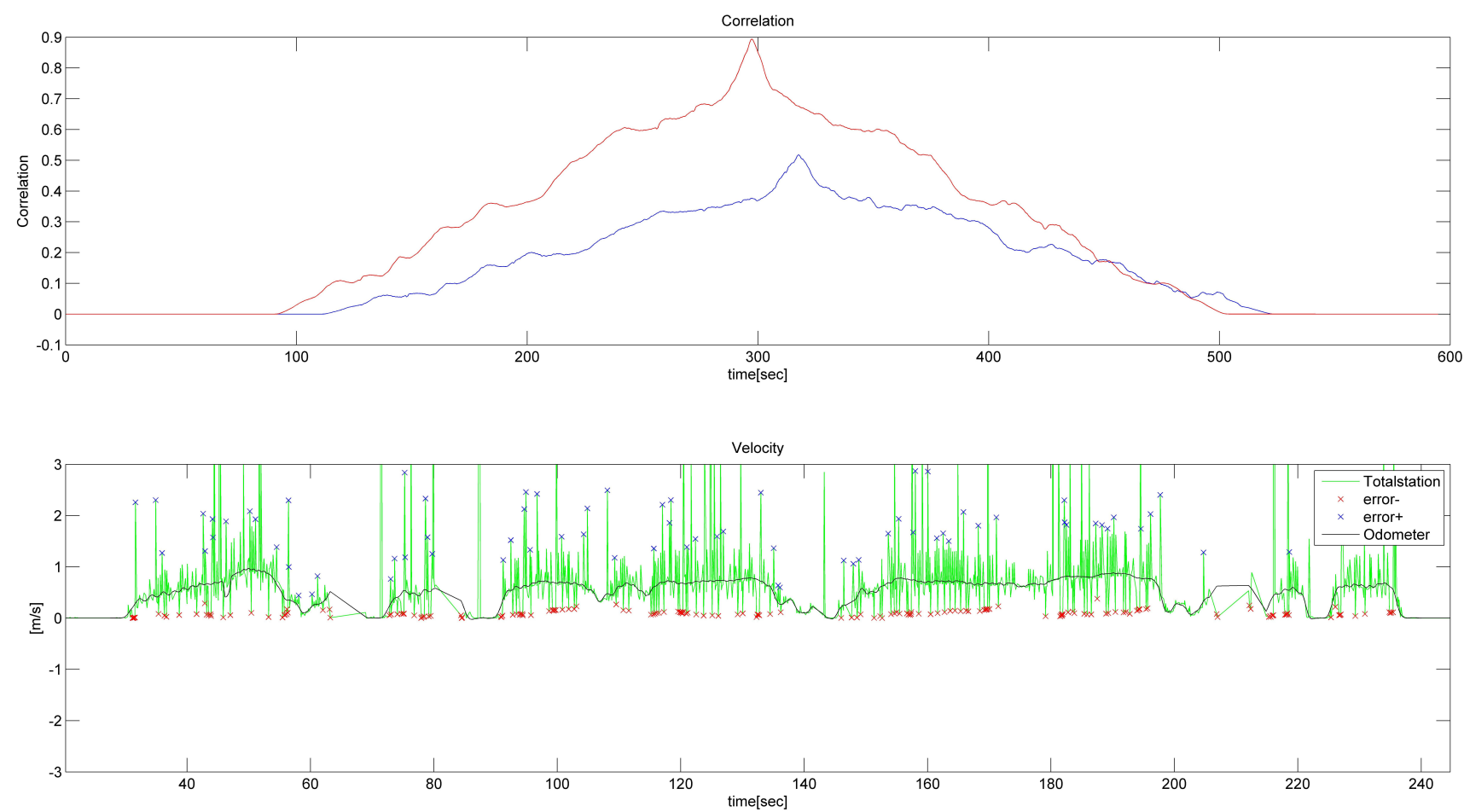
It seems that the trajectory conforms well to the measurements from the IMU and odometer. However, if the solution is reviewed again in more detail, the time offset can clearly be determined through the correlation calculation (Figure 11). The calculated velocities from the total station data were subject to large scatter. Consequently, the velocity profiles of the total station and odometer do not match.

The correlation coefficient of the blue curve is correspondingly small, namely $51 \%$ (Figure 11 ). It is interesting that the points labeled by the total station as code 1284 and the points classified as "OK" both suffered this problem. To filter out the incorrect measurements, the deviation of the odometer velocity was calculated using the first correlation calculation from each velocity. All the measurements with a deviation higher than $50 \%$ were removed. With the remaining $78 \%$ of the measurements, the correlation calculation was increased to $89 \%$ (red curve).

Table 3. Quadratic deviation of control points; Case 4.

\begin{tabular}{ccc}
\hline & All (m) & Adjusted (m) \\
\hline Maximum & 0.5719 & 0.0958 \\
Median & 0.0101 & 0.0098 \\
Mean & 0.0186 & 0.0157 \\
Std. & 0.0410 & 0.0174 \\
\hline
\end{tabular}

Then, the trajectory was estimated with these data by using Kalman filtering. Only every second point determined by the total station was used for control purposes. Figure 12 and Table 3 show the coordinate differences between the filter solution and the control points. In Figure 12, one can clearly observe certain abnormal behaviors other than the white noise, which has to be discussed.

Figure 12. Coordinate differences between total station control points and the Kalman filter from trajectory 2. The variations in $\mathrm{X}$ and $\mathrm{Y}$ are presented in red and blue, respectively. It is striking that the differences are greater in the track direction.

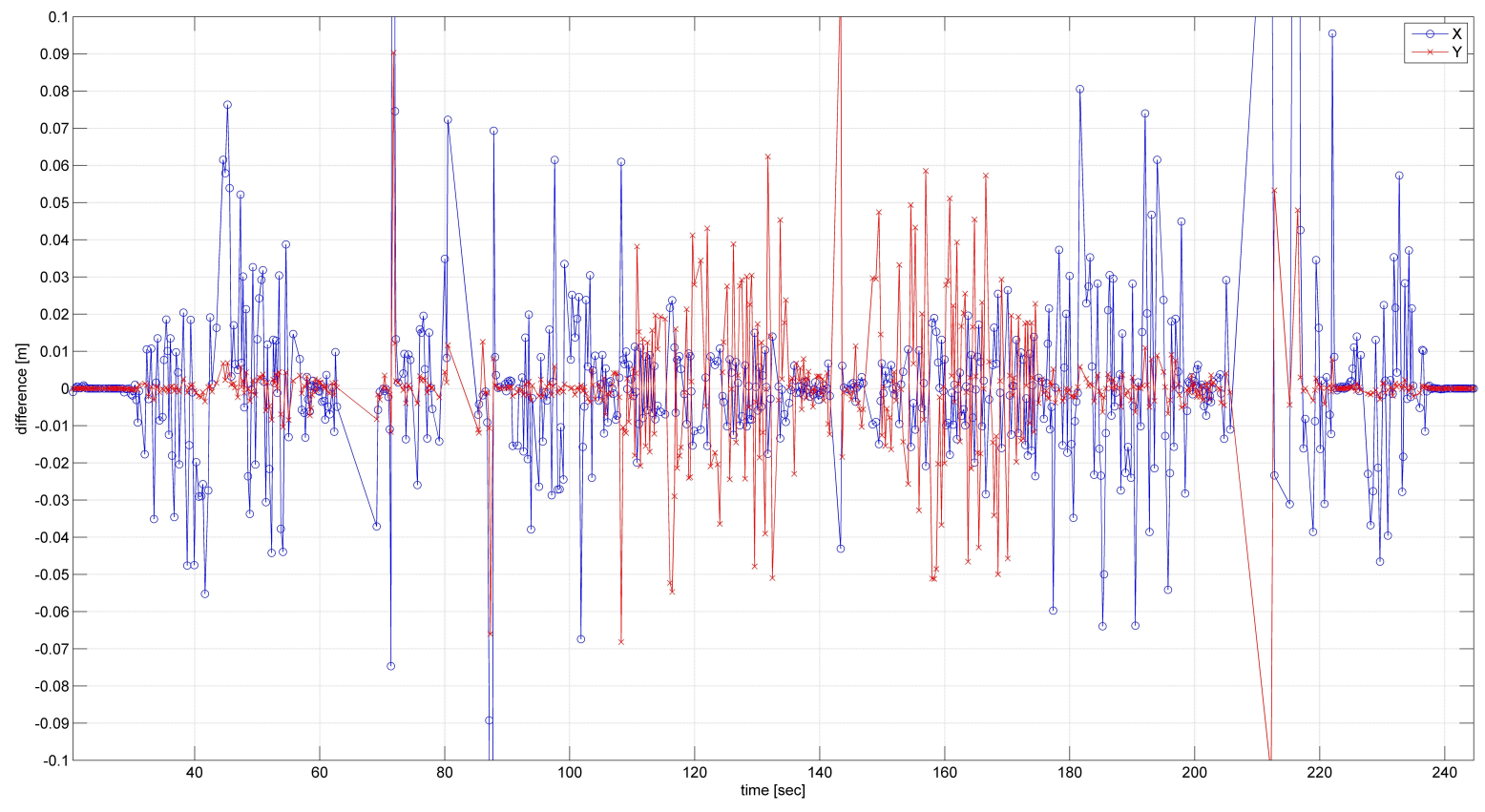


The moving direction of the platform during the test was mainly in the X-direction (east). After it crossed the starting point, the platform moved in the Y-direction (north). A strong correlation between the direction of motion and the magnitude of the coordinate differences could be observed. The coordinate differences are clearly significant along the track direction of the trajectory, but are reasonably small along the cross-track direction. The cause of this phenomenon is still under investigation. On the one hand, this may have resulted from an incorrect time apportionment, because the time could have a much greater impact on the trajectory estimation along the track direction than along the cross-track direction. On the other hand, the distance measurements from the total station can be problematic. This problem also affects the along-track direction, due to the location used. The deviations shown in Table 3 are slightly higher than those in Case 3 , when all measurements were considered. However, with the GeoCom interface, a significantly high data rate could be produced. In Case 3, the measuring frequency was $1 \mathrm{~Hz}$, as the data rate in Case 4 was, on average, $8 \mathrm{~Hz}$. At a higher data rate, the plausibility of the measurements can be checked. Movements of more than $10 \mathrm{~cm}$ within $100 \mathrm{~ms}$ are unlikely to result in a maximum speed of $1 \frac{\mathrm{m}}{\mathrm{s}}$. If these measurements are not removed, a deviation of $1.5 \mathrm{~cm}$ could occur.

Finally, a laser scanning scene recorded indoors by the used system is shown in Figure 13. The estimated trajectories from the in-house developed Kalman filter were combined with the scanned data. Then, the transformation of all the scanned points was performed using a specifically developed program. The results were satisfactory. The plane objects showed deviations within $>10 \mathrm{~cm}$, but if the point cloud is considered in greater detail, errors are clearly visible. When the total station's aiding module is exposed, there may be cracks.

Figure 13. Images (a-c) show a point cloud measured with IMU, scanner and total station. (a) Overview of trajectory 1, as in Figure $8 \mathrm{a}$; (b,c) show that a generally good point cloud could be created; (c) shows some irregularities.

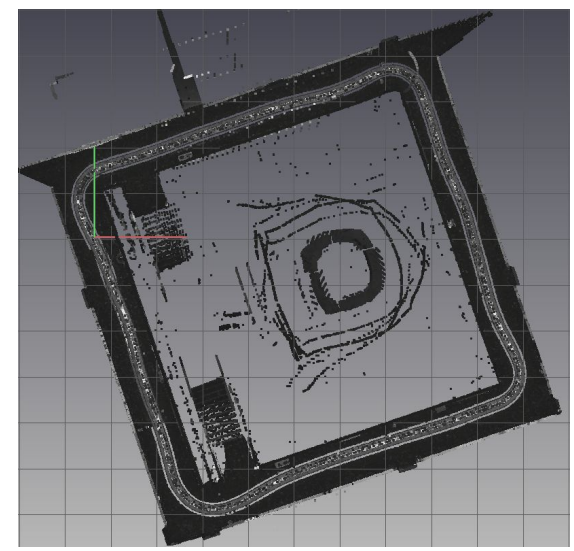

(a)Overview

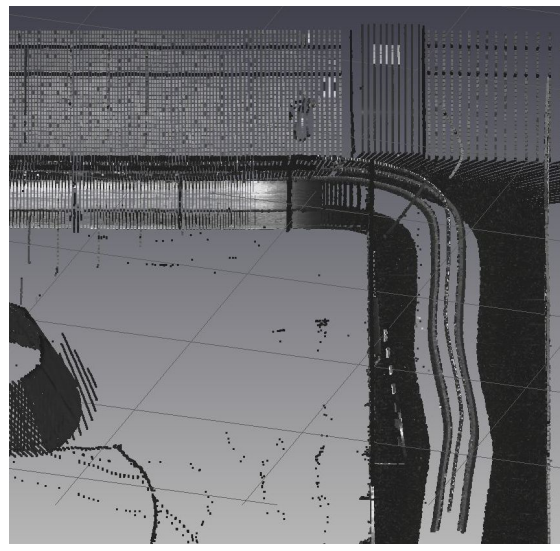

(b)Detail 1

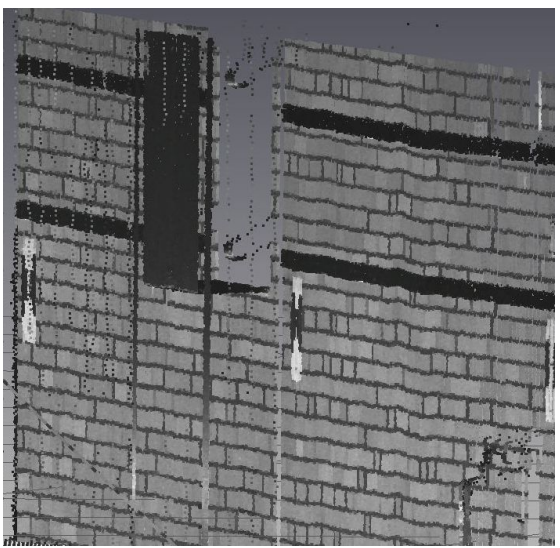

(c)Detail 2

\section{Conclusions}

In this paper, the question was whether it is possible to use a mobile mapping system in the interior of buildings. The biggest problem here is the absence of GNSS. For this purpose, a suitable replacement is sought. This is necessary to prevent the drift of the system. In this paper, it was shown that this 
replacement with a total station can be delivered. Difficulties arise as a result of the time synchronization of both systems. The cross-correlation is a useful tool for estimating the offset of the different time systems. However, the full potential of this tool has not yet been exhausted. With the approach presented here, however, at least one of the trajectory accuracies can be achieved, which is comparable to GNSS. The tests have shown that the examination is no longer possible with GNSS. The next step is to find a suitable examination for the measuring system to verify the results. A solution is found with the laser tracker, as it offers both high temporal and spatial resolution. Control via GeoCom still has not yielded the desired improvement. However, the results are very promising, with the exclusion of obvious measurement error problems. In this case, an approach should be developed for automatic outlier detection. For the measurement platform, however, the preliminary results already represent the first success. In summary, it can be ascertained that a quick survey of indoor areas is possible using an indoor mobile mapping system. The loss of GNSS can be compensated for, by using a total station. In order to expand the system, it will be necessary to develop new modules. After the upcoming improvement of the module "total station", a stereo camera system is integrated into the complete system as the next module. In the next step, opportunities of simultaneous localization and mapping are investigated by means of one or several laser scanners.

\section{Conflict of Interest}

The authors state that they have no conflict of interest.

\section{References}

1. El-Sheimy, N. An Overview of Mobile Mapping Systems. In Proceedings of the FIG Working Week 2005, Cairo, Egypt, 16-21 April 2005.

2. Kaartinen, H.; Hyyppä, J.; Kukko, A.; Jaakkola, A.; Hyyppä, H. Benchmarking the performance of mobile laser scanning systems using a permanent test field. Sensors 2012, 12, 12814-12835.

3. Brenner, C. Extraction of features from mobile laser scanning data for future driver assistance systems. Adv. GISci. 2009, doi: 10.1007/978-3-642-00318-9_2.

4. Haala, N.; Peter, M.; Kremer, J.; Hunter, G. Mobile LiDAR mapping for 3D point cloud collection in urban areas-A performance test. Int. Arch. Photogr. Remote Sens. Spat. Inf. Sci. 2008, 37, 1119-1127.

5. Hassan, T.; El-Sheimy, N. Common adjustment of land-based and airborne mobile mapping system data. Int. Arch. Photogramm. Remote Sens. Spat. Inf. Sci. 2008, 37, 835-842.

6. Puente, I.; González-Jorge, H.; Martínez-Sánchez, J.; Arias, P. Review of mobile mapping and surveying technologies. Measurement 2013, 46, 2127-2145.

7. Petrie, G. An introduction to the technology mobile mapping systems. Geoinformatics 2010, 13, $32-42$.

8. Graham, L. Mobile mapping systems overview. Photogr. Eng. Remote Sens. 2010, 76, 222-228.

9. Graefe, G. Quality Management in Kinematic Laser Scanning Applications. In Proceedings of the The 5th International Symposium on Mobile Mapping Technology (MMT’07), Padua, Italy, 29-31 May 2007. 
10. Schwarzenberger, J. Evaluierung und Kalibierung eines Mobile Mapping Systems. M.Sc. Thesis, HafenCity Universität, Hamburg, Germany, 2013.

11. International Hydrographic Organization (IHO). Chapter 3 Depth Determination. In Manual on Hydrography Publication C-13, 1st ed.; International Hydrograpic Organization, International Hydrographic Bureau: Monaco, China, 2011.

12. Keller, F.; Sternberg, H. Kombination von Hydrographie und Terrestrischen Laserscannern-Systematische Effekte, Kalibrier- und Auswertemethoden. In Proceedings of Bundesanstalt für Gewässerkunde: Kolloquium Neue Entwicklungen in der Gewässervermessung, Koblenz, Germany, 20-21 November 2012.

13. Seube, N.; Picard, A.; Rondeau, M. A simple method to recover the latency time of tactical grade IMU systems. ISPRS J. Photogramm. Remote Sens. 2012, 74, 85-89.

14. Stempfhuber, W.; Schnaedelbach, K.; Maurer,W. Genaue Positionierung von bewegten Objekten mit Zielverfolgenden Tachymetern. In Proceedings of the XIII International Course on Engineering Surveying (Ingenieurvermessung 2000), München, Germany, 13-17 March 2000.

15. Kalman, R.E. A new approach to linear filtering and prediction problems. ASME J. Basic Eng. 1960, 82, 35-45.

16. Welch, G.; Bishop,G. An Introduction to the Kalman Filter; Technical Report 95-041; University of North Carolina Computer Science-Chapel Hill: Chapel Hill, NC, USA, 1995.

17. Rauch, H.E.; Tung, F.; Striebel, C.T. Maximum likelihood estimates of linear dynamic systems. AIAA J. 1965, 3, 1445-1450.

18. Grewal, M.S.; Andrews, A.P. Kalman Filtering. In Theory and Practice Using MATLAB; Wiley: Hoboken, NJ, USA, 2008.

19. Beetz, A. Separation of Control Quality and Measurement Accuracy for Guiding Control Tasks of an Indoor Construction Machine Simulator. In Proceedings of International Conference on Indoor Positioning and Indoor Navigation (IPIN 2012), Sydney, Australia, 13-15 November 2012.

20. Leica. Leica TPS1200+ Leica TS30/TM30 GeoCOM Reference Manual; Version 1.50; Leica Geosystems AG: Heerbrugg, Switzerland 2009.

(C) 2013 by the authors; licensee MDPI, Basel, Switzerland. This article is an open access article distributed under the terms and conditions of the Creative Commons Attribution license (http://creativecommons.org/licenses/by/3.0/). 